\title{
El suavizado exponencial en el pronóstico de series no estacionarias.
}

\section{The exponential smoothing in the forecast of non-stational series.}

Mercedes del Carmen Franco Rodríguez. ${ }^{1}$ \& Efraín Velasteguí López. ${ }^{2}$

Recibido: 10-11-2019 / Revisado: 01-12-2019 /Aceptado: 22-12-2019/ Publicado: 04-01-2020

\begin{abstract}
.
DOI: https://doi.org/10.33262/cienciadigital.v4i1.1073

Tourism is one of the most dynamic sectors of the Cuban economy and one of its main sources of income. As of May 2019, the arrival of visitors to Cuba has begun to slow down as a result of the resurgence of the blockade. The immediate effects of the enactment of Title III of the Helms Burton Act have caused a slowdown in the growth in the number of visitors and income in recent months. In statistical terms, these effects translate into a change of trend in the series of visitors that are also characterized by their marked seasonality. The study addresses econometric techniques for the seasonally adjusted series and the search for short-term forecasts that guarantee the minimum error. Exponential Smoothing is used specifically Holt-Winters Multiplicative Model to project the behavior of the arrival of visitors. The forecasts of the series are an important tool for the decision makers in the sector that face a dynamic and highly uncertain environment.
\end{abstract}

Keywords: Time series, exponential smoothing, trend, Seasonality, tourism.

\section{RESUMEN}

El turismo es uno de los sectores más dinámicos de la economía cubana y una de sus principales fuentes de ingreso. A partir del mes de mayo del 2019 el arribo de visitantes a Cuba ha comenzado a desacelerarse como resultado del recrudecimiento del bloqueo.

\footnotetext{
${ }^{1}$ Doctora en Ciencias Económicas. Profesora Titular de la Facultad de Turismo de la Universidad de la Habana, la Habana Cuba., mercedes.franco@ftur.uh.cu

${ }^{2}$ Doctor en Ciencia Pedagógicas, Director, Editor del consocio Ciencia Digital, Ambato, Ecuador., luisefrainvelasteguio@ cienciadigital.org
} 
Los efectos inmediatos de la puesta en vigor del Título III de la Ley Helms Burton han provocado una desaceleración del crecimiento del número de visitantes y de los ingresos en los últimos meses. En términos estadísticos estos efectos se traducen en un cambio de tendencia en la serie de visitantes que se caracteriza además por su marcada estacionalidad. El estudio aborda técnicas econométricas para la desestacionalización de la serie y la búsqueda de pronósticos en el corto plazo que garanticen el mínimo error. Se utiliza el Suavizado exponencial específicamente el Modelo Multiplicativo Winter para proyectar el comportamiento del arribo de visitantes. Los pronósticos de la serie son una importante herramienta para los decisores del sector que se enfrentan a un entorno dinámico y de gran incertidumbre.

Palabras clave: Series cronológicas, suavizado exponencial, tendencia, estacionalidad, turismo.

\section{Introducción.}

El proceso de transformación del Modelo Económico Cubano contempla el sector del turismo como una de sus prioridades. Apostando por que la actividad turística tenga un crecimiento acelerado que dinamice la economía, logrando eslabonamientos productivos y el incremento de las utilidades. Respondiendo a estas pautas en los últimos años en el sector se ha crecido extensivamente en el número de habitaciones, Caballero (2017).

Como respuesta a esta política se han reportado incrementos en el número de visitantes por año, alcanzando en el 2018 la cifra record de 4711910 (Oficina Nacional de Estadística e Información, 2019). Cuba creció rápidamente en las plataformas de intermediación y búsqueda como Airbnb entre los años 2016 y 2018 generando incrementos en los ingresos de la actividad turística y un impulso en el Sector No estatal que llegó a generar en el 2017 el $21,4 \%$ del total de los ingresos.

Sin embargo, a partir del mes de mayo del 2019 el arribo de visitantes ha comenzado a desacelerarse; este descenso es un efecto al recrudecimiento del bloqueo. El gobierno norteamericano ha endurecido los requisitos para que los estadounidenses puedan viajar a Cuba, sus medidas han limitado las posibilidades de inversión, de comercio y de transporte hacia y desde Cuba. La empresa de tecnología transnacional alemana con matriz estadounidense Trivago eliminó a Cuba de sus sistemas de búsqueda y reservación a causa de las restricciones impuestas por EUA. Los efectos inmediatos de la puesta en vigor del Título III de la Ley Helms Burton han provocado una desaceleración del crecimiento del número de visitantes y de los ingresos en los últimos meses.

En el 2018 los ingresos totales disminuyeron en 333, 6 millones de cuc, contrayéndose en el 2018 con relación al 2017 en un 10\%. Al analizar la composición del ingreso total el Sector No Estatal Cubano ha sufrido una desaceleración más significativa disminuyendo sus 
ingresos en el 32,9\%, mientras que el Ingreso por transporte Internacional ha sufrido la drástica disminución de un 43, $26 \%$.

Tabla 1. Visitantes a Cuba e Ingresos anuales del sector en el período 2012-2018.

\begin{tabular}{lcccc}
\hline Año & $\begin{array}{l}\text { Visitantes } \\
\text { (unidades) }\end{array}$ & $\begin{array}{l}\text { Ingresos Totales } \\
\text { (millones } \\
\text { cuc) }\end{array}$ & $\begin{array}{l}\text { Ingresos del Sector No } \\
\text { Estatal } \\
\text { (millones de cuc) }\end{array}$ & $\begin{array}{l}\text { Ingresos por Transporte } \\
\text { Internacional } \\
\text { (millones de cuc) }\end{array}$ \\
\hline 2012 & 2851 & $2613,3^{* *}$ & $358,3^{* *}$ & $287,7^{* *}$ \\
& $616^{* *}$ & & & \\
2013 & $2862273^{*}$ & $2607,8^{*}$ & $365,0^{*}$ & $282,7^{*}$ \\
2014 & $3013584^{*}$ & $2546,1^{*}$ & $378,9^{*}$ & $178,8^{*}$ \\
2015 & $3540175^{*}$ & $2818,6^{*}$ & $485,2^{*}$ & $217,8^{*}$ \\
2016 & $4009169^{*}$ & $3068,6^{*}$ & $537,3^{*}$ & $161,5^{*}$ \\
2017 & $4653559^{*}$ & $3301,7^{*}$ & $706,6^{*}$ & $115,8^{*}$ \\
2018 & $4711910^{*}$ & $2968,1^{*}$ & $473,9^{*}$ & $65,7^{*}$ \\
\hline
\end{tabular}

Fuente: Elaborado por los autores a partir de *Anuario Estadístico de Cuba 2018. Turismo. Edición 2019, Oficina Nacional de Estadística e Información y **Anuario Estadístico de Cuba 2017. Turismo. Edición 2018, Oficina Nacional de Estadística e Información.

Este escenario desfavorable impone a Cuba el reto de ser un destino competitivo e insertarse en nuevos mercados. Para el diseño de estrategias que le permitan mejorar los ingresos es imprescindible el estudio de las series cronológicas relacionadas con la actividad. A partir de los métodos estadísticos para el tratamiento de las series puede definirse cuál es el pronóstico de visitantes para los próximos meses y por tanto los ingresos que pueden esperarse.

\section{Desarrollo.}

El buen comportamiento de los negocios depende de factores externos como la evolución de la economía, la estabilidad política, los problemas financieros y depende también de factores internos: necesidades de insumos, estrategias financieras de inversión, búsqueda de mercado, autofinanciación y otros que deben ser tenidos en cuenta y previstos por toda empresa que quiere ser efectiva. El comportamiento de estos factores que pueden influir en el éxito o no de un negocio, puede ser previsto mediante el estudio de series de tiempo o series cronológicas.

Una serie temporal es el resultado de observar valores de una variable a lo largo del tiempo en intervalos regulares (días, años, meses). La representación gráfica de una serie de tiempo "habla" de sus características más importantes. El análisis de series permite la descripción detallada de una variable determinada y la predicción del futuro. La variante clásica del 
estudio univariado de una serie considera el estudio de cuatro componentes que la caracterizan: tendencia, estacionalidad, ciclo o cíclico y aleatorio.

Las series de tiempo poseen características que las distinguen de los datos de corte transversal:

- El orden de las observaciones es relevante: cronológico y único.

- Se parte del supuesto de que existe correlación serial: el valor de la serie en el instante t está correlacionado con el valor del período t-1.

Las series temporales pueden clasificarse de alta o baja frecuencia. Se consideran series de alta frecuencia aquellas que se expresan en horas, días, minutos. Estas se distinguen por una media estable y no presentan estacionalidad. Su varianza cambia con el tiempo con periodos de alta y baja volatilidad. Por su parte las series de baja frecuencia se expresan usualmente en meses, trimestres, y sus características más comunes son la presencia de tendencia, estacionalidad y varianza, que crece con la media.

Las series suelen clasificarse en estacionarias cuando la media y la varianza son constantes a lo largo del tiempo. Gráficamente se observa cuando los valores de la serie tienden a oscilar alrededor de la media constante con variabilidad constante. Las no estacionarias son aquellas en las que la tendencia o variabilidad cambian en el tiempo. En estos casos los cambios en la media determinan una tendencia a crecer o decrecer a largo plazo, los valores no oscilan alrededor de un valor constante.

Los objetivos al modelar una serie pueden ser: capturar la tendencia y el comportamiento estacional observado, tratar la varianza no constante (heterocedasticidad) o modelar la autocorrelación serial.

Al modelar una serie puede conseguirse un modelo univariante o un modelo de relación. Los modelos univariantes explican la correlación de una variable temporal usando para ello su propia historia pasada y reciente. Los pronósticos de estos modelos funcionan muy bien en el corto plazo. Por su parte los modelos de relación utilizan modelos de regresión con el objetivo de predecir en el corto y en el largo plazo.

Una serie temporal puede ser descrita mediante la siguiente expresión:

$Y_{t}=T_{t}+S_{t}+C_{t}+\varepsilon_{t}$

Donde:

$Y_{t}$ : valor de la serie.

$T_{t}$ : tendencia.

$S_{t}$ : estacionalidad.

$C_{t}:$ componente cíclico. 
$\varepsilon_{t:}$ error.

Los supuestos son:

$E\left[\varepsilon_{t}\right]=0, \forall t \quad E\left[\varepsilon^{2} t\right]=\sigma^{2}, \forall \mathrm{t}$

$E\left[\varepsilon_{t} \varepsilon_{s}\right]=0, \quad \forall t \neq s \quad \varepsilon \sim N\left[0, \sigma^{2}\right]$

Cuando se quiere explicar la tendencia de la serie los modelos más simples son:

$$
\begin{array}{ll}
T_{t}=\alpha_{0}+\alpha_{1} T, & \forall t=1,2, \ldots, n \\
T_{t}=\alpha_{0}+\alpha_{1} T+\alpha_{2} T^{2}, & \forall t=1,2, \ldots, n
\end{array}
$$

El estimado de la serie para el período $\mathrm{t}+\mathrm{k}$ vendría dado por:

$$
\hat{Y}_{t}(k)=\hat{\alpha}_{0}+\hat{\alpha}_{1}(t+k)
$$

Donde $\hat{\alpha}_{0}$ y $\hat{\alpha}_{1}$ son los parámetros estimados.

Si la serie no presenta estacionalidad estos modelos, aunque son útiles para describir el comportamiento de la serie, presentan limitaciones cuando se utilizan para efectuar pronósticos ya que solo son capaces de reproducir las pautas de la serie ofreciendo estimaciones con altos errores cuadráticos asociados.

Otra forma que puede utilizarse para capturar los cambios en media (tendencia creciente, decreciente, etc) y los cambios en la varianza (crece la dispersión conforme crece la media), consiste en realizar transformaciones en los datos que eliminen esas características. La transformación logarítmica hace que la dispersión sea constante a medida que crece la media, pero no consigue una tendencia constante (estacionaria en media). Las series estacionarias en media y varianza son más fáciles de modelizar.

En este estudio contamos con la serie de baja frecuencia expresada en meses-años. La información disponible son los visitantes a Cuba en el período enero 2009 - agosto 2019, que incluye 128 observaciones. Los datos fueron obtenidos de los Anuarios Estadísticos que publica la Oficina Nacional de Estadística e Información de Cuba (ONEI) y de Servicios Informativos que aparecen en su página web. La serie será desestacionalizada utilizando el alisado o suavizado exponencial.

\section{Resultados.}

El tratamiento de las series temporales ha evolucionado a métodos más precisos, que permiten lograr mejores pronósticos a partir de todos los elementos que contiene la serie. Este estudio se planteó como objetivo presentar un pronóstico para la serie de visitantes a Cuba para el año 2019, mediante las técnicas de suavizado exponencial; específicamente el 
Modelo Multiplicativo de Winter. Para tratar la serie se buscarán las variaciones estacionales y las constantes de alisamiento exponencial que minimicen los errores cuadráticos.

El análisis del gráfico de secuencia de la serie permite visualizar la existencia de tendencia creciente hasta el 2018 y el cambio del componente $T$ en los últimos meses correspondientes al período enero-agosto del 2019. La estacionalidad (S) se manifiesta en forma de dientes de serrucho alrededor del movimiento general de la serie. La serie es no estacionaria, es evidente la presencia de la estacionalidad.

Figura 1. Gráfico de Secuencia de la serie de arribo de visitantes enero 2009-agosto 2019.

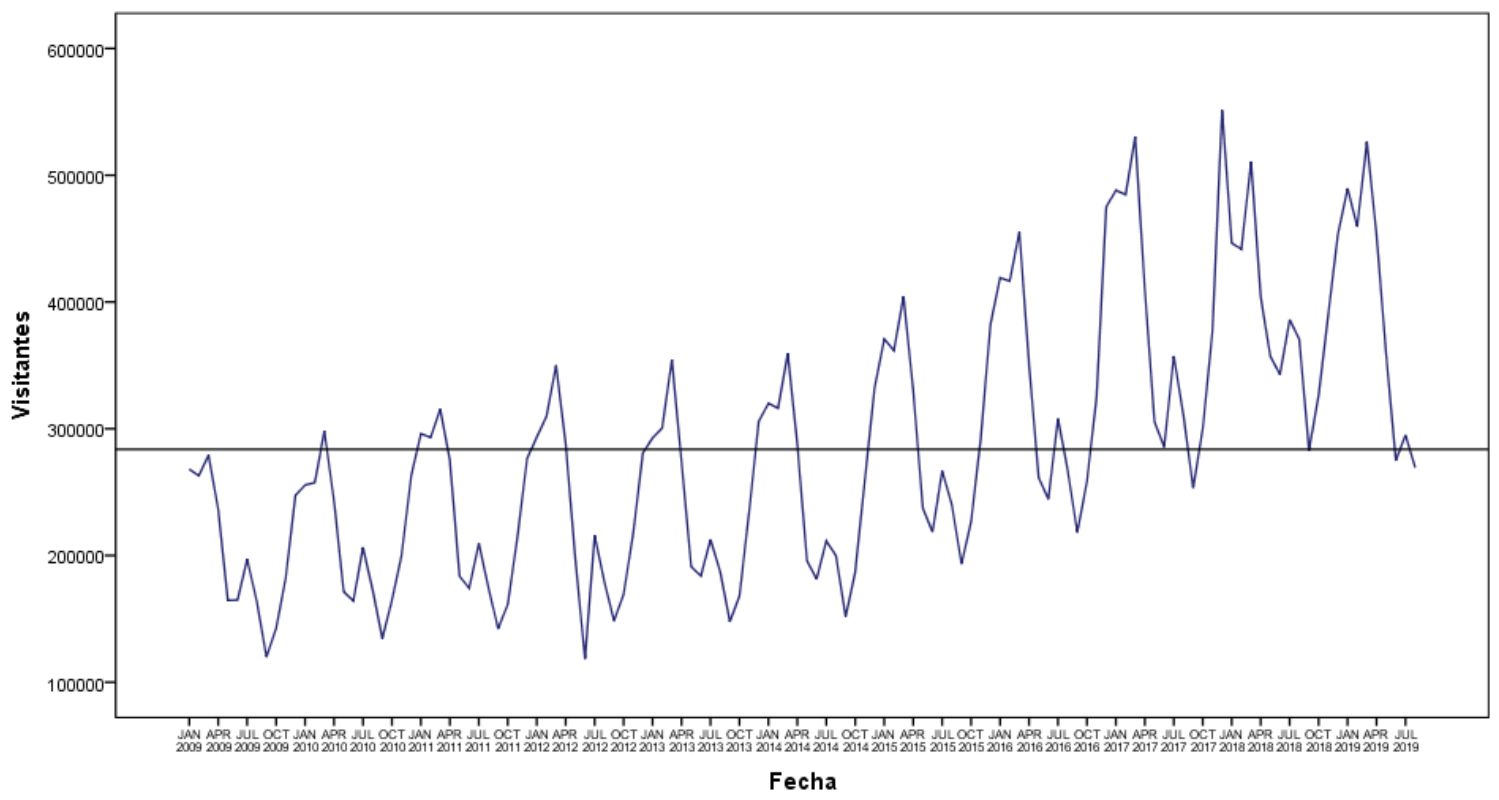

Fuente: Elaborado en software IBM SPSS Statistics 20 a partir de los datos del Anuario Estadístico de Cuba 2019 (ONEI).

Los índices estacionales permiten apreciar los cambios que se producen al interior de la serie en periodos menores de un año. No son más que promedios de variaciones de los valores reales de la serie para cada estación p. Estos pueden ser calculados por modelos aditivos o multiplicativos, los primeros explican los cambios con relación a la tendencia de la serie en unidades y los segundos en $\%$.

$S_{j}=\frac{\sum_{j=1}^{p} \frac{Y_{j}}{\bar{Y}_{j}}}{p}$ 
Donde:

$\mathrm{S}_{\mathrm{j}}$ : variación estacional en la estación j

p: cantidad de periodos o estaciones.

$Y_{j}$ : valor real de la serie correspondiente al período $\mathrm{j}$.

$\widehat{Y}_{j}$ : valor estimado de la serie correspondiente al período $\mathrm{j}$.

Al realizar la descomposición estacional de la serie haciendo uso del software IBM SPSS Statistics 20.0 se obtienen los siguientes resultados.

Tabla 2. Factores estacionales (\%) para la serie Visitantes a Cuba en el período 20092019.

\begin{tabular}{lllllllllllll}
\hline Mes & 1 & 2 & 3 & 4 & 5 & 6 & 7 & 8 & 9 & 10 & 11 & 12 \\
\hline $\begin{array}{l}\text { Factor } \\
\text { estacion }\end{array}$ & 129, & 128, & 143, & 115, & 82, & 75, & 93, & 79, & 62, & 73, & 94, & 122, \\
al & & 8 & 5 & 1 & 8 & 0 & 4 & 8 & 3 & 2 & 9 \\
\hline
\end{tabular}

El factor estacional calculado por el modelo multiplicativo muestra los meses donde la serie se comporta por encima del valor de la tendencia de la serie y viceversa. Corroborando que el periodo de mayor arribo de visistantes es enero-abril y el mes de diciembre. El conocimiento del patrón estacional permite estimar las variaciones que se van a producir en los próximos meses, a partir de estos pronósticos las entidades turisticas pueden anticiparse y definir nuevas estrategías de comercialización.

El proceso de desestacionalización de la serie permite obtener una ecuación que refleje más nítidamente la tendencia de la serie, que la obtenida si seguimos el procedimiento con la serie original, que contiene periodicidades que no permiten apreciar claramente la tendencia. Como resultado de la destacionalización se crean cuatro variables como se aprecia en la figura 2.

- SAF_1: representa las variaciones estacionales por el modelo multiplicativo en \%.

- ERR_1: representa los errores o sea los cocientes entre la serie desestacionalizada SAS_1 y la variable STC_1.

- STC_1: Es el ciclo de tendencias representa la serie desestacionalizada entre el error STC_1=SAS_1/ERR_1

- SAS_1: serie desestacionalizada, SAS_1=Y $*$ SAF_1 a la serie original por la variación estacional. 
Figura 2. Componentes de la serie visitantes en el período enero 2009-agosto 2019.

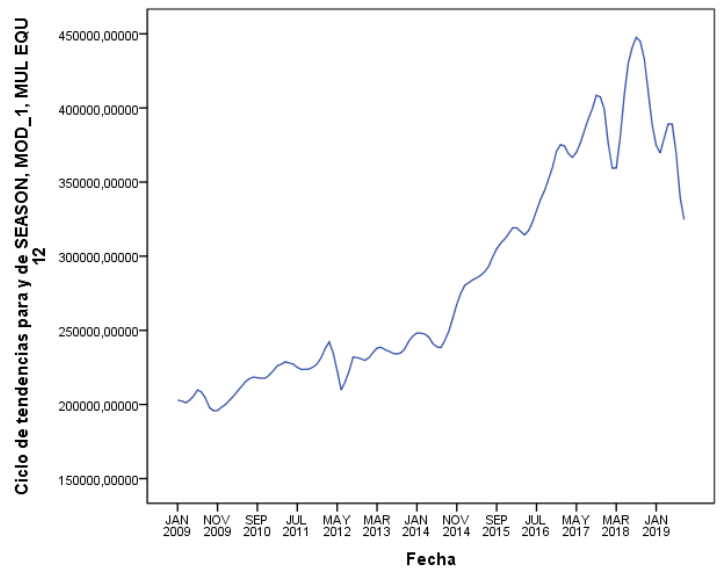

STC_1: Serie Tendencia ciclo

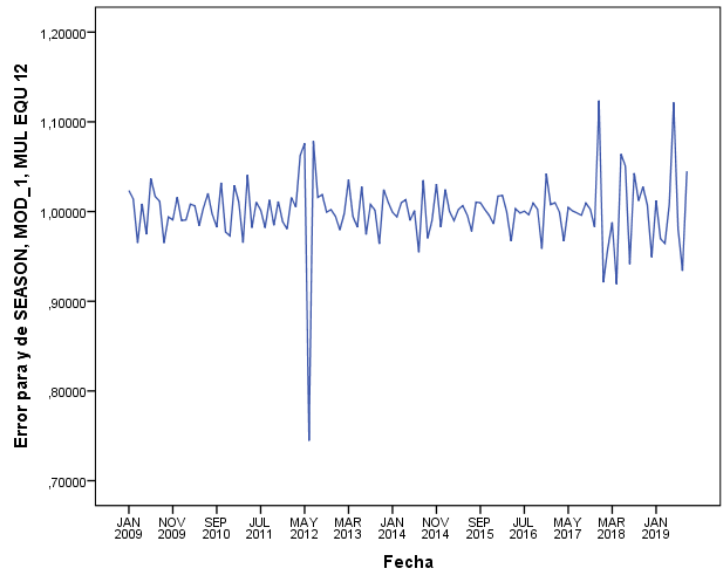

ERR_1: Componente irregular

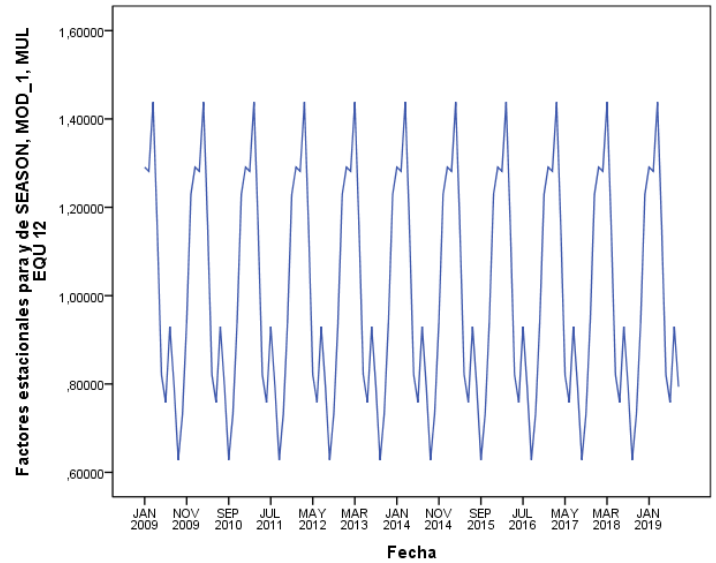

SAF_1: Factores estacionales

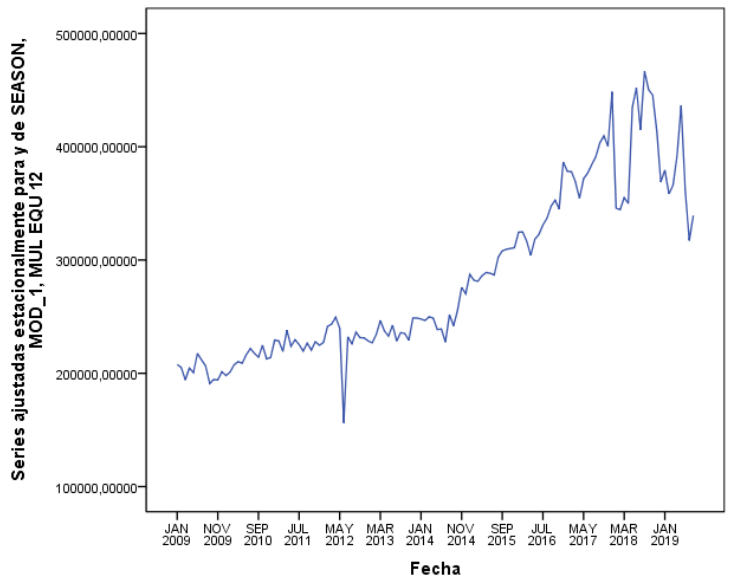

SAS_1: Serie desestacionalizada

Cuando se quiere relacionar dos series a través de una ecuación de regresión, resulta más apropiado trabajar con la información de ambas series desestacionalizadas, lo que suministrará resultados más precisos. A partir de la serie desestacionalizada SAS_1 puede hacerse un análisis de regresión para estimar la tendencia de la serie y volver a incorporar el patrón estacional. Pero está solución no reproduciría el cambio de tendencia en el arribo de visitantes que se ha producido en los meses transcurridos del 2019.

A continuación, se muestran las representaciones gráficas de la serie original visitantes y la ajustada a partir de calcular la tendencia haciendo uso de la estimación curvilínea. A pesar 
de que la ecuación de tendencia de la serie es significativa, los pronósticos obtenidos como se muestra en la figura distan de los valores reales.

Figura 3. Serie original y ajustada de visitantes en el período enero 2009-agosto 2019.

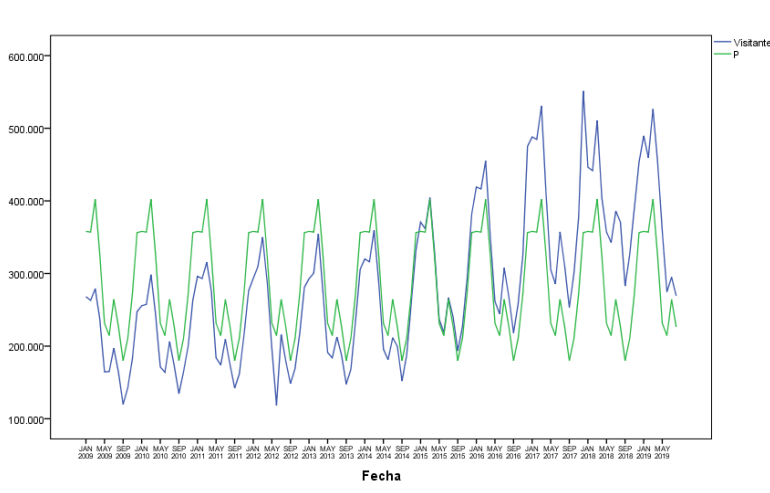

Pronóstico de la serie

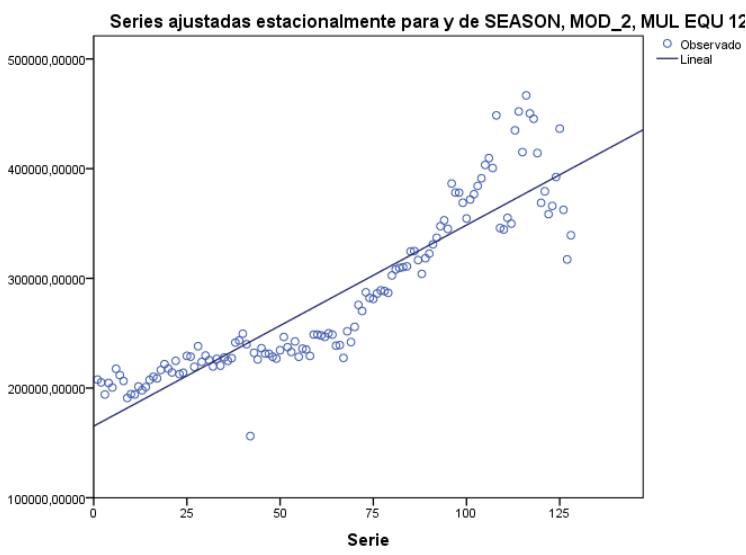

Tendencia estimada por estimación curvilínea

Tabla 3. Resumen del Modelo de estimación curvilínea para la tendencia.

Variable dependiente: Series ajustadas estacionalmente para y de SEASON, MOD_2, MULEQU 12

\begin{tabular}{|l|r|c|r|r|r|r|c|}
\hline & \multicolumn{4}{|c|}{ Resumen del modelo } & \multicolumn{3}{c|}{$\begin{array}{c}\text { Estimaciones de los } \\
\text { parámetros }\end{array}$} \\
\cline { 2 - 10 } Ecuación & R cuadrado & $\mathrm{F}$ & gl1 & gl2 & Sig. & Constante & b1 \\
\hline Lineal &, 827 & 604,168 & 1 & 126 &, 000 & 165275,467 & 1833,093 \\
\hline
\end{tabular}

Una variante de pronóstico adecuada en esta situación es el Alisamiento exponencial este método introducido por Brown (1963) puede utilizarse con dos fines:

- Obtener una serie suavizada, al estilo de las medias móviles.

- Realizar pronósticos de la serie.

Este método genera pronósticos acertados para muchas variables de series de tiempo y reconoce el impacto decreciente de períodos cada vez más lejanos en el pasado. Esta característica del método permite incorporar de manera confiable un cambio de tendencia como el que se ha producido en los últimos meses en el arribo de visitantes a Cuba. Esa suavización se hace expresando el pronóstico de cada año como una combinación ponderada de dos cantidades: el valor de la variable real en el período anterior y el pronóstico hecho para ese año de la variable.

Las razones que han hecho popular el uso del alisamiento exponencial son la poca cantidad de datos que se requiere, y el hecho de que la mayoría de los softwares incluyen módulos de 
alisamiento. Es una técnica extremadamente simple de entender y aplicar. Los nuevos pronósticos se derivan ajustando los pronósticos previos, y los pronósticos pueden revisarse continuamente basándose en experiencias previas. Entre las ventajas que ofrece el método de Alisamiento Exponencial se encuentran:

- Se encuentra incorporado en múltiples softwares estadísticos.

- Requieren un mínimo de información comparado con otros métodos de pronósticos.

- El método permite incorporar en el pronóstico los cambios en el patrón de los datos.

- Existe la posibilidad de dar mayor peso a las primeras o a las últimas observaciones, en dependencia del interés del investigador.

La constante de alisamiento se denota como $\alpha$ y es un valor que está comprendido entre $(0<$ $\alpha<1$ ). Cuando $\alpha$ está cercana a 1, la información más reciente afectará más al nuevo pronóstico. Cuando el valor de $\alpha$ es cercano a 0 , el nuevo pronóstico será muy parecido a la observación más antigua.

En el alisado exponencial, los pesos asignados a los valores observados disminuyen al paso del tiempo, por lo que cuando se hacen cálculos, el valor observado más reciente recibe el peso más alto, el valor observado con anterioridad, recibe el segundo valor más alto y así sucesivamente, recibiendo el peso más bajo el valor observado inicialmente.

Para obtener un suavizado de la serie $\mathrm{y}_{1}, \mathrm{y}_{2}, \ldots, \mathrm{y}_{\mathrm{n}}$ se utiliza la siguiente expresión:

$$
\hat{Y}_{t+1}=\alpha Y_{t}+(1-\alpha) \hat{Y}_{t}
$$

Donde:

$\mathrm{Y}_{\mathrm{t}}$ : valor de la serie en estudio para el período $\mathrm{t}$.

$\hat{Y}_{t}$ : valor estimado de la serie para el período t.

$\hat{Y}_{t+1}$ : valor alisado (suavizado de la serie en el período $t+1$ )

$\alpha$ : constante de alisamiento.

Así mientras más alto es $\alpha$ es más sensible el pronóstico a los cambios del valor reciente de Y, y el alisado será menor. Y mientras más cercano este $\alpha$ de cero, será mayor el alisamiento. Un $\alpha$ pequeño se puede usar con datos de series de tiempo que son relativamente estables. $\mathrm{Si}$ la serie cambia rápidamente $\alpha$ debe ser grande, porque asigna pesos grandes a las observaciones más recientes.

La determinación de $\alpha$ generalmente está basada en el valor que minimice la Suma de los Cuadrados de los residuos. Actualmente IBM SPSS Statistics 20 muestran como resultados aquel alisado que garantice el mínimo error de estimación. 
En el caso de la serie en estudio es apropiado el uso del suavizado exponencial con el modelo Multiplicativo de Holt-Winters, ya que se ha comprobado con anterioridad su marcado patrón estacional. Eso implica que se incluirían tres constantes de alisamiento; Alpha $(\alpha)$ para el movimiento general de la serie, Ganma $(\gamma)$ para la tendencia y Delta $(\delta)$ para la estacionalidad.

El método de Holt-Winters utiliza las siguientes fórmulas:

$$
\begin{gathered}
Y_{t}=\alpha Y_{t}+[1-\alpha]\left[Y_{t-1}+T_{t-1}\right] \\
T_{t}=\gamma\left[Y_{t}-Y_{t-1}\right]+[1-\gamma]\left[T_{t-1}\right] \\
E_{t}=\delta\left[Y_{t}-Y_{t-1}\right]+[1-\delta]\left[E_{t-s}\right] \\
F_{t+1}=\left[Y_{t}+T_{t}\right] E_{t-s+1}
\end{gathered}
$$

Donde:

$\alpha$ : constante de alisamiento de nivel.

$\gamma$ : constante de alisamiento para la tendencia.

$\delta$ : constante de alisamiento para la estacionalidad.

$\mathrm{Y}_{\mathrm{t}}$ : valor alisado de la serie en estudio para el período $\mathrm{t}$.

$E_{t-s+1}$ : estimación de la estacionalidad en el período t-s+1

$T_{t}$ : es la estimación de la tendencia en el periodo t.

$F_{t+1}$ : es el pronóstico para el período t.

El modelo ajustado tiene un $\mathrm{R}^{2}=0.95$ y las significaciones asociadas a los parámetros significativas para Alpha y Delta. El parámetro Ganma no es significativo no obstante en estos casos no se invalida el modelo por una constante de alisamiento puede tomar valor cero.

La figura 4 muestra la serie ajustada y los límites de confianza para el estimado construidos con un $95 \%$ de confiabilidad.

Tabla 4. Parámetros del Modelo Multiplicativo de Winter.

\begin{tabular}{|lll|r|r|r|r|}
\hline Modelo & & Estimación & \multicolumn{1}{c|}{ ET } & \multicolumn{1}{c|}{ S } & Sig. \\
\hline Visitantes-Modelo_1 & Sin transformación & Alpha (Nivel) &, 496 &, 072 & 6,906 &, 000 \\
& & Gamma (Tendencia) &, 003 &, 010 &, 253 &, 801 \\
& & Delta (Estación) &, 541 &, 130 & 4,165 &, 000 \\
\hline
\end{tabular}


Figura 4. Serie ajustada de visitantes en el período enero 2009 - septiembre 2020.

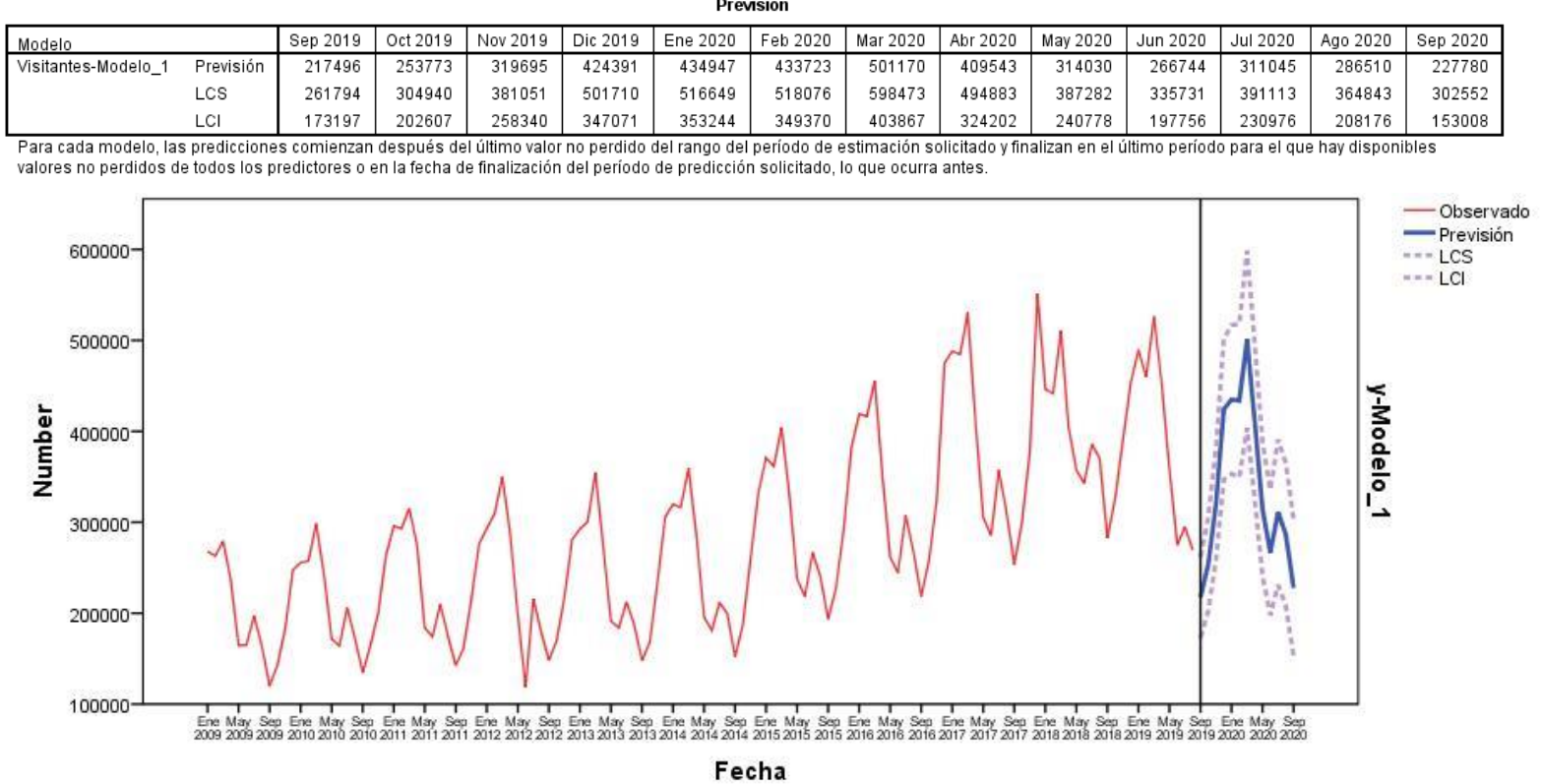

Como resultado del alisamiento exponencial podemos estimar que este año el número total de visitantes estará entre los 4107286 y los 4575566 con una confiabilidad del 95\%. Lo que implica un cambio en la tendencia anual de los últimos 10 años.

Tabla 5. Estimados para el año 2019.

\begin{tabular}{lccc}
\hline Mes & Visitantes & $\begin{array}{c}\text { Límite inferior del intervalo } \\
\text { de confianza (LCI) }\end{array}$ & $\begin{array}{c}\text { Límite superior del intervalo } \\
\text { de confianza (LCS) }\end{array}$ \\
\hline Enero & 489618 & 489618 & 489618 \\
Febrero & 459417 & 459417 & 459417 \\
Marzo & 526422 & 526422 & 526422 \\
Abril & 453104 & 453104 & 453104 \\
Mayo & 358321 & 358321 & 358321 \\
Junio & 274837 & 274837 & 274837 \\
Julio & 295042 & 295042 & 295042 \\
Agosto & 269310 & 269310 & 269310 \\
Septiembre & $217496^{*}$ & $173197^{*}$ & $261794^{*}$ \\
Octubre & $253773^{*}$ & $202607^{*}$ & $304940^{*}$ \\
Noviembre & $319695^{*}$ & $258340^{*}$ & $381051^{*}$ \\
Diciembre & $424391^{*}$ & $347071^{*}$ & $501710^{*}$ \\
Total & $4341426^{*}$ & $4107286^{*}$ & $4575566^{*}$ \\
\hline
\end{tabular}


*Estimados a partir del alisamiento exponencial por el Modelo Multiplicativo de Winter

\section{CONCLUSIONES}

- Generar estimaciones y el cuerpo de indicadores y variables que faciliten a los decisores definir estrategias y políticas para el turismo es imprescindible. El uso de herramientas econométricas permite realizar pronósticos vitales en el escenario dinámico e incierto en el que se desarrolla la economía mundial.

- Los autores mediante el Modelo Multiplicativo Winter han proyectado el comportamiento del arribo de visitantes a Cuba, estimando la llegada de 4107286 a 4575566 visitantes en el 2019 con un error probable del 5\%.

- En los últimos años se ha apostado por un crecimiento extensivo del turismo en Cuba priorizando el incremento del número de visitantes, los pronósticos obtenidos sugieren repensar está política priorizando el uso eficiente de las capacidades existentes y la diversificación de los mercados emisores.

- Este método para el tratamiento de series temporales es recomendable sobre todo en los estudios de las series turísticas caracterizadas por su marcada estacionalidad. La proyección de la cantidad de visitantes posibilita estimar haciendo uso de modelos de regresión los ingresos del sector y otras variables de interés.

\section{BIBLIOGRAFIA}

1. Aguilar. R, Garcia. A, Vidal. P (2009). Elementos de Econometría. Aplicaciones para Cuba.

2. Anderson, T. W. (1971). The statistical analysis of time series. Hohn Wiley \& Sons. New York.

3. Anuario Estadístico de Cuba 2017 (2018). Oficina Nacional de Estadística e Información.

4. Anuario Estadístico de Cuba 2018 (2019). Oficina Nacional de Estadística e Información.

5. Box, G.E.P; Jenkis, G.M; Reinsel, G.C. (1994). Time series analysis: Forecasting and control. (Third Edition) Prentice Hall.

6. Brown, R. (1963). Smoothing, forecasting and prediction of discrete time series. PrenticeHall. New Jersey.

7. Caballero, P; Rosales, S; Alfonso, G (2017). Economía Cubana: Desafíos de su desarrollo. Instituto Nacional de Investigaciones Económicas (INIE).

8. Gras, J. A. (Ed.). (2001). Diseños de series temporales: técnicas de análisis (Vol. 46). Edicions Universitat Barcelona.

9. Mauricio, J. A. (2007). Análisis de series temporales. Universidad Complutence de Madrid.

10. Peña, D. (1989). Estadística (Modelos y Métodos 2): Modelos Lineales y Series Temporales (Segunda Edición), Alianza Editorial. 
11. Pérez, C. (2006). Problemas Resueltos de Econometría. Universidad Complutense de Madrid, Thomson.

12. Wooldridge, J.M (2006). Introducción a la Econometría. Un enfoque moderno, (2nd edition), Thomson.

\section{PARA CITAR EL ARTÍCULO INDEXADO.}

Franco Rodríguez, M. del C., \& Velasteguí López, E. (2020). El suavizado exponencial en el pronóstico de series no estacionarias. Ciencia Digital, 4(1), 97-110. https://doi.org/10.33262/cienciadigital.v4i1.1073

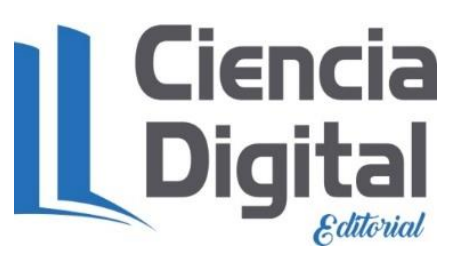

El artículo que se publica es de exclusiva responsabilidad de los autores y no necesariamente reflejan el pensamiento de la Revista Ciencia Digital.

El artículo queda en propiedad de la revista y, por tanto, su publicación parcial y/o total en otro medio tiene que ser autorizado por el director de la Revista Ciencia Digital.
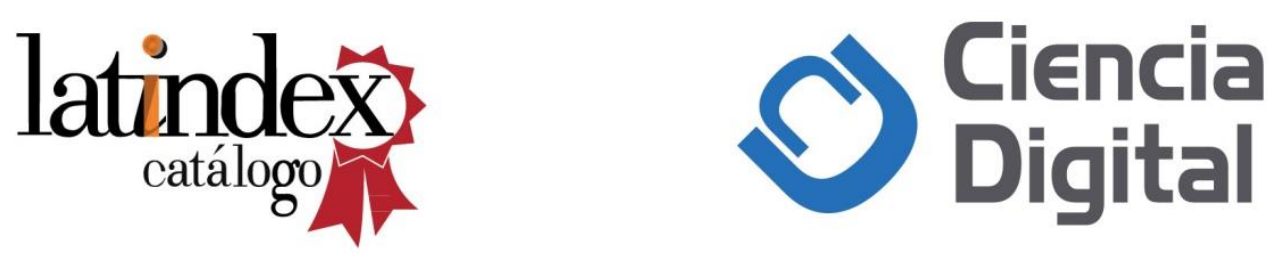